\title{
Experimental Models of Integrative Healthcare in a Regional Health System: A Complex Innovation Path
}

\author{
Francesco Cardini $^{\mathrm{a}}$ Giulia Guerzoni ${ }^{\mathrm{a}}$ Corrado Ruozi ${ }^{\mathrm{a}}$ \\ Healthcare and Social Agency of Emilia Romagna Region, Bologna, Italy
}

Developing experimental models of integrative healthcare is 1 of the 6 research priorities in the roadmap for CAM [1,2] clinical research in Europe setup by the CAMbrella project (FP7 HEALTH 2009, GA No. 241951). The term 'complex' in the title of this editorial refers to both the innovation represented by the introduction of CAM modalities and approaches in conventional healthcare routines and the path (strategies and course) to be covered in order to achieve that goal.

In Emilia Romagna (a north-eastern Italian region with a resident population of 4.46 million) where the health system has the dual statutory task of providing healthcare and doing research, the regional government policy regarding CAM has been inspired by the principle of considering CAM interventions as possible innovations to be integrated in the normal prevention and care pathways after an experimental phase of use and the consequent evaluation of impact.

In other Italian regions different policies regarding CAM have been promoted, variously positioned between 2 extreme radical political choices: ignoring the phenomenon of large CAM use within the population and entirely leaving it to the private sector (CAM available only for people who can pay for it, outside the regional health system (RHS)), or including a number of CAM modalities in the RHS, free of cost or at an accessible cost, with dedicated service, structure, and staff as any other conventional specialty. The first approach inevitably generates or confirms an inequality as it devolves CAM to the market as the main regulator; in this way CAMs remain a social phenomenon with high cultural and economic impact, but limited interactions/interrelation with conventional medicine. The second approach entails an equitable, but blind, offer of various CAMs (starting from acupuncture, homeopathy, and phytotherapy) without or before a rational evaluation of their impact on citizens' health, and puts considerable public resources into consolidating structures, practices, and expectations that could be difficult to reform in case of observed low, null, or negative impact on citizens' health. The third (experimental) way, chosen by the government of the Emilia Romagna Region, seems to be a more rational policy, able, at least in theory, to avoid the risk (implied in both of the above mentioned approaches) of reducing healthcare from a right to a commodity [3], and to respect the basic principles of the RHS, i.e. equity, accessibility, and sustainability. On the other hand, the implementation of this policy requires facing a number of complicated issues.

In fact, in order to be included in the system, an ideal 'new' intervention/approach should be safe, effective, feasible and generalizable within the RHS clinical settings, accepted by patients and health professionals, and sustainable in terms of costs. At present, this amount of information is available only for few CAM treatments/approaches, and scarce resources are available for realizing good quality clinical research in this field. In other words, the RHS would need to select the innovations to be integrated in the routine healthcare, but, in the CAM field, selection is biased by the scarce quantity and quality of the available evidence.

Another difficulty is the need for mixed methods research for promoting and evaluating the integration process. In fact, integration cannot suddenly happen because of an administrative decision, as it is and should be conceived as 'an individual and societal process of change, which develops in the mind and behavior of patients, providers and other stakeholders in different contexts of care'[4]. From this point of view, integrative healthcare, rather than a pre-defined entity, is an objective to be pursued in an experimental way, through the setup and evaluation of models.

What is a model of integrative healthcare? The following definition has been adopted in 2012 by the Observatory on Unconventional Medicines of Emilia Romagna Region (OMncER; a board of experts of conventional and unconventional medicine nominated by the regional government in

\section{KARGER \\ Fax +4976145207 14 \\ Information@Karger.com}

www.karger.com
(C) 2014 S. Karger GmbH, Freiburg

$1661-4119 / 14 / 0212-0084 \$ 39.50 / 0$

Accessible online at:

www.karger.com/fok
Francesco Cardini, MD

Healthcare and Social Agency of Emilia Romagna Region

Viale Aldo Moro 21, 40127 Bologna, Italy

fcardini@regione.emilia-romagna.it 
Table 1. Set of methods available for researching models of integrative healthcare

\begin{tabular}{ll}
\hline Evaluation of & Suitable research methods \\
\hline Effectiveness & $\begin{array}{l}\text { comparative effectiveness studies (pre-post case series, pragmatic RCT, comparison } \\
\text { between services, etc.) } \\
\text { large observational studies } \\
\text { Safety }\end{array}$ \\
$\begin{array}{l}\text { Patients' and providers' knowledge, motivations, } \\
\text { preferences, attitudes }\end{array}$ & $\begin{array}{l}\text { qualitative research methods (case reports, narrations, interviews, focus group, } \\
\text { Delphi process, consensus conference, etc.) }\end{array}$ \\
$\begin{array}{l}\text { Cultural and operational difficulties of the } \\
\text { integration process }\end{array}$ & $\begin{array}{l}\text { qualitative research methods (see above, in particular: focus group) } \\
\text { health services research (survey) } \\
\text { methods for promoting change (action research, participatory research) } \\
\text { quality improvement (audit) }\end{array}$ \\
costs observation, services comparison, cost-effectiveness studies
\end{tabular}

order to support the implementation of the regional policy regarding $\mathrm{CAM}$ ) in order to describe the research topic of the 3rd Unconventional Medicines Experimental Program of Emilia Romagna Region: 'A model of integrative healthcare is a potentially generalizable organizational modality of interaction between providers, between services, and between providers and services, resulting in the coordinated supply of conventional and unconventional (CAM) treatments/procedures appropriate for prevention and/or care of a specific health condition. The model of integrated healthcare can include single treatments or packages of treatments in the routine diagnostic and care pathways; it has to be developed as a research project and evaluated on the basis of pre-planned quantitative and qualitative outcomes' [5].

In simpler words, a model of integrative healthcare should set up the prevention and care pathways offered to the patients who face a particular health problem; the key concepts in the above definition are: interaction, coordination, potential generalizability, and experimental approach. 2 points must be underlined: first, the main focus of the integration process is the patient and his/her health condition, not the promotion or evaluation of 1 or more CAM modalities; second, research on integrative healthcare models is a complex matter that requires complex methods.

The set of methods needed for studying models of integrative healthcare has been discussed by the OMncER and is reported in table 1 . This table is included in a policy document written and approved by the OMncER in December 2011 and available (in Italian) online [6]. It can be noticed that, beside quantitative research methods frequently employed in medicine, methods more common in humanistic studies (qualitative research) and methods to reach consensus and implementing changes are also included.

The inventory of methodological tools included in table 1 can help to choose the most suitable for different research questions. The resulting body of knowledge should include and allow to evaluate what we must not lose on the way doing research, i.e. the strong and innovative points of CAM, able to favor the change to a better healthcare for all; understanding of the patient as an inseparable mind-body unity; individualized approach; focus on 'salutogenesis' (health production and conservation) beside and beyond diseases' treatment; attention to patient's preferences and to cultural and context factors; complexity of any intervention, never to be seen as only technical.

Following the addresses of its policy document, in 2012 the OMncER worked intensively setting up the III CAM Experimental Program, by prioritizing the clinical conditions on which to develop experimental models of integrated care; selecting the CAM treatments/modalities worth including in such models on the basis of their considerable amount of evidences of safety and effectiveness; and identifying other CAM treatments/modalities with interesting preliminary data or other features (e.g., high prevalence of use) that make them worth studying as well.

In particular, 3 OMncER work groups (chronic non-cancer pain, patients with cancer, women's health) selected the core research topics of the 3rd Regional Experimental Program, using 4 main criteria: relevance and prevalence of the health problem, availability of safe and effective CAM interventions for that health problem, experience accumulated in the still ongoing 2nd CAM Regional Experimental Program, and feasibility in the RHS of Emilia Romagna. The selected core research topics are about the 'setup and evaluation of models of integrative healthcare for: - treatment of chronic non-cancer pain (particularly headache and low back pain) by Acupuncture; support and treatment of labor pain by Acupressure; - quality of life and prevention of adverse effects of conventional treatments by Viscum Album in patients with cancer'. It must be underlined that acupuncture, acupressure, and V. album will be the 'pillars' of the respective projects because of the relative solidity of the available evidences in literature, but also other CAM treatments, less proven but, for example, highly prevalent, can be studied as 'satellites' of the main project.

After the call for participation in May 2013, the interested RHS Local Healthcare Units nominated the competent clinicians/researchers in charge for participating in 3 working groups that will be in charge of the project protocols; the OMncER will coordinate, support, and supervise the process. 
Table 2. Promoting and misleading attitudes and behaviors along the path toward integrative healthcare

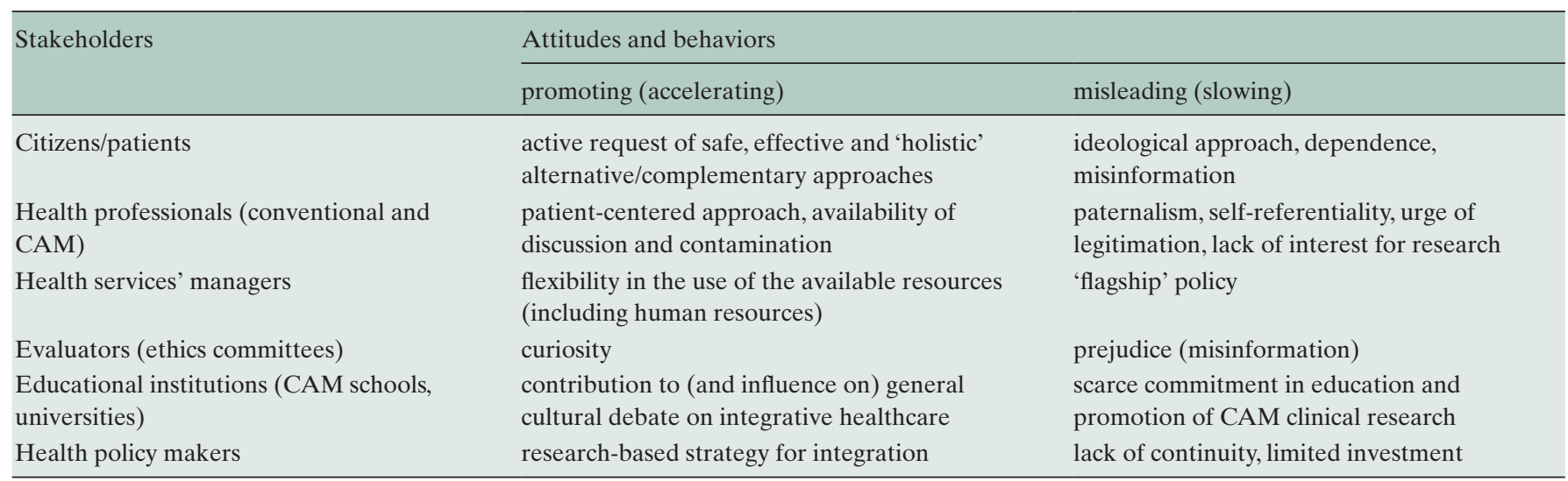

The 3 working groups met in June 2013 and started to work on the projects that will be financed by the government of Emilia Romagna Region, and implemented by regional research networks of local health units, after the approval of the competent ethics committees. It is expected that representatives of all relevant stakeholders (including patients and RHS executives) will revise and contribute to the protocols.

As stated at the very beginning of this editorial, and as can be gathered from the description above, the involvement of a RHS in an experimental program aimed to setup, implement, and evaluate the impact of integrative healthcare models is a complicated issue. Despite the fact that the process in Emilia Romagna is still in a very initial phase, a number of strengths and fragilities of such an institutional approach are already evident and can be highlighted.

\section{Points of Strength}

It is a rational, prudent and transparent approach, which tries to translate research into clinical practice as well as generalize the best available evidence, and promotes dialogue and collaboration between health professionals with different medical cultures and background in a framework of pragmatic, patient-centered, and independent (publicly funded) research. The peer dignity of all actors on the scene and the institutional legitimation give solidity to the whole process which occurs in the 'real world' and not in artificial settings.

\section{Points of Fragility}

This 'consensus-based' approach is inevitably slow; despite formally independent (institutionally promoted, transparently funded, conflict of interests-free), it is exposed to the pressures of both favorable and adverse lobbies or individuals inside and outside of the RHS; political changes, such as elections or changes in staff (quite frequent in the RHS), can waste the work done or hamper the work in course; the present economic crisis limits the already scarce available funds; but for sure, the main difficulty is that health professionals fully and heavily engaged in clinical work have difficulties in finding time for conducting good quality clinical research.

The only way to face and overcome these fragilities is to sustain the entire process with a strong and efficient centralized regional infrastructure that provides methodological support, accurate supervision and monitoring as well as continuous education and information for all interested parties (citizens/patients and health professionals). Table 2 describes a number of promoting and misleading attitudes and behaviors of the main stakeholders along the path toward integrative healthcare.

Looking at this (surely incomplete) list, it is self-evident that, without a determined and balanced cultural initiative of education and information, the chances of success of such a complex enterprise as research on models of integrative healthcare are limited.

\section{References}

1 Fischer F, Lewith G, Witt CM, et al.: A research roadmap for complementary and alternative medicine - what we need to know by 2020. Forsch Komplementmed 2014;21:e1-e16.

2 Fischer F, Lewith G, Witt CM, Linde K, von Ammon K, Cardini F, Falkenberg T, Fønnebø V, Johannessen H, Reiter B, Uehleke B, Weidenhammer W, Brinkhaus B: High prevalence but limited evidence in complementary and alternative medicine: guidelines for future research. BMC Complement Altern Med 2014;14:46.

\footnotetext{
3 No authors listed: The NHS: free and caring or a market commodity? Lancet 2013;382:571.

4 Weidenhammer W, Brinkhaus B: CAMbrella - a panEuropean research network for complementary and alternative medicine: from the beginnings up to first results. Forsch Komplementmed 2012;19(suppl 2):3-5.

5 Area Sviluppo delle professionalità per l'assistenza e la salute. http://assr.regione.emilia-romagna.it/it/aree attivita/sviluppo-professionalita/mnc-1/eventi-segnalazioni/ecim-2012/5th-european-congress-for-integrative-medicine-ecim-2012 (accessed 06.03.2014).
}

6 Area Sviluppo delle professionalità per l'assistenza
e la salute. http://assr.regione.emilia-romagna.it/it/
servizi/pubblicazioni/rapporti-documenti/f072-per-un-
programma-sperimentale-regionale-sulla-integrazione-
delle-medicine-non-convenzionali-nei-percorsi-as-
sistenziali-del-servizio-sanitario (accessed 06.03.2014).

Cardini/Guerzoni/Ruozi 